\title{
Least Square Method for inferring Phylogenic trees
}

\section{Método do quadrado mínimo para inferir árvores filogênicas}

DOI: $10.46814 / 1 a j d v 4 n 2-001$

Recebimento dos originais: 04/02/2022

Aceitação para publicação: 01/03/2022

Felipe Fernandes Albrecht

PhD Doktors der Naturwissenschaften (Dr. rer. nat.))

Institution: F. Hoffmann-La Roche

Address: Lehenmattstrasse, 301, 4052 Basel Suíça

E-mail: felipe.albrecht@gmail.com

\section{Antônio Luís Sombra de Medeiros}

Mestrado em Modelagem Matemática - Fundação Getúlio Vargas

Institution: Pontifical Catholic University of Rio de Janeiro - PUC-Rio (Pontifícia Universidade

Católica do Rio de Janeiro - PUC-Rio)

Address: Rua Lauro Muller 26, apt. 306 - Botafogo - Rio de Janeiro/RJ - Brasil, CEP: 22290160

E-mail: aluis.852@gmail.com

\section{Mitri Martins Alves de Britto}

Graduação Engenharia Elétrica

Institution: Instituto Militar de Engenharia (IME)

Address: Rua Guararapes 469 apt. 83 - 04561-000 - Brooklin - São Paulo / SP

E-mail: britto.mitri@gmail.com.br

Nelson Antônio Borges Garcia

Doutor em Matemática Aplicada - ECL - Lyon -França (1986)

Institution: Instituto Militar de Engenharia (IME)

Address: Rua das Palmeiras, 93 apt. 208 - 22270-070 - Botafogo - Rio de Janeiro/RJ

E-mail: nborgesster@gmail.com

\begin{abstract}
Nowadays, an important problem in Biomathematics is how to efficiently compute the length for the phylogenic tree branches. The approach followed herein is to employ the distance matrices method which leads to least square problems. The related tree construction strategy then generates block tridiagonal systems of linear equations. Experiments in solving them with the conjugate gradient method coupled to different block preconditioners were performed. Preliminary results confirm a high performance for some preconditioning choices.
\end{abstract}

Keywords: least squares method, linear system, preconditioned conjugate gradient method, phylogenic tree. 


\section{RESUMO}

Atualmente, um problema importante em Biomatemática é como calcular eficientemente o comprimento dos galhos filogênicos das árvores. A abordagem seguida aqui é empregar o método de matrizes de distância que leva a problemas menos quadrados. A estratégia de construção de árvores relacionada gera então sistemas tridiagonais em bloco de equações lineares. Foram feitas experiências para resolvê-las com o método de gradiente conjugado acoplado a diferentes pré-condicionadores de blocos. Os resultados preliminares confirmam um alto desempenho para algumas escolhas de précondicionamento.

Palavras-chave: método dos mínimos quadrados, sistema linear, método do gradiente conjugado précondicionado, árvore filogênica.

\section{INTRODUCTION}

Molecular phylogenies describe the study of evolution relationships between living beings, protein and genetic sequences and other molecular taxons. Inferring phylogenies from molecular data may be carried out through the point analysis of similarities and differences in the studied sequences. One class of molecular phylogenies is the distance matrices method, with a matrix whose entries represent the distinctions between the taxons under study. According to Felsenstein [6], the Distance Matrices Methods may shortly describe as: "compute a distance estimate for each pair of species and then search a tree that better approaches the distance set". The procedure discards all data originated from any combination bearing a high number of characters and the reduce the data matrix associated to pairs of distances. In recent years there has been increased interest in producing large and accurate phylogenic trees using some numerical computational methods approach. To construct large and accurate trees for a large number of taxons, the computational processing increases and computational methods can be helpful, see Keane and Stamatakis $[9,10]$. We work with the distance matrix methods for inferring phylogenies by taking a large number of taxons. These methods have been introducing by Cavalli- Sforza and Edwards [5] and by Fich and Margoliash [7]. The methods introduced by CavalliSforza and Edwards [5] use a set of linear equations where the number of equations and their variables grow up with the number of taxons, linearly. Here, we use efficient computational methods to solve the numerical block linear systems associated to the length of the branches of the phylogenic trees.

\section{MOTIVATION}

The least square method is not suitable to get from the distance matrices the sides length for each branch of the considered trees. For a given tree topology, the solution of the corresponding system of linear algebraic equations, see by Cavalli- Sforza and Edwards [5], becomes intractable with direct methods if we are interested in a high number of taxons. The matrix associated to the least-squares 
method, being symmetric and positive definite, allows the use of the conjugated gradient method (CGM). We know that in the worst-case convergence is guaranteed in $\mathrm{n}$ steps, being $\mathrm{n}$ the linear system order. Since each iteration for the CGM requires $n 2+O(n)$ multiplications and divisions, we deduce that in this case we need $n 3+O(n 2)$ operations, which is the same amount needed for a complete inversion of the matrix with the LLt decomposition. In order to improve convergence for CGM, we get hold of the matrix profile. It turns out that the matrices that appear are all block-matrices, being all blocks also symmetric and positive definite. We can then use the Preconditioned Conjugate Gradient method with block Jacobi as a preconditioner - this amounts to incomplete block decomposition. It is known that block Jacobi method has a rather slow convergence but, when employed as a preconditioner, overall convergence is speeded up.

\section{THE METHOD}

Given T, a tree without root and having a set of taxons L, our aim is to find a set of distances between these taxons in such a way that these distances turn out to be the best approximations - in the least-squares sense - to the observed distances. The measure given by the least-squares method has the expression:

$$
\mathrm{Q}=\sum_{i=1}^{n} \sum_{j=1, j \neq i}^{n} w_{i j}\left(D_{i j}-d_{i j}\right)^{2}
$$

In (1), $d_{i j}$ denotes the sum of the length of the branches connecting taxons $i$ to $j, D_{i j}$ means the distances given by the distance matrix while $w_{i j}$ stands

for the weights, here taken equal to unity. Also, Q means for the sum of the squares of the differences between Dij, the distances given by the tree entries, and dij, the inferred distances.

The least-squares method aims to minimize the value for $\mathrm{Q}$. This minimum is reached first taking the derivatives of $\mathrm{Q}$ with respect to the branch's length, then making the resulting equation all equal to zero. It is seen that this system solution minimizes the value for $\mathrm{Q}$.

The first step consists in generating the matrix $\mathrm{X}$ to represent the position the taxons occupy in the tree in a matrix representation. Here each column represents a side in the tree, while each row represents a pair of taxons. If the path between two taxons $\mathrm{i}$ and $\mathrm{j}$ passes through branch $\mathrm{k}$, we must put label 1 on line ij and column $\mathrm{k}$, otherwise we label this position with 0 . This coeficiente is denoted by $\mathrm{xij}, \mathrm{k}$. 
In equation (1) coefficients dij denotes the sums of the branches' length. We expect that the distance between $\mathrm{i}$ e $\mathrm{j}$ is:

$$
d_{i j}=\sum_{k} x_{i j, k} v_{k}
$$

Where vk represents the length of the k-th branch. Consequently equation (1) becomes:

$$
\mathrm{Q}=\sum_{i=1}^{n} \sum_{j=1, j \neq i}^{n} w_{i j}\left(D_{i j}-\sum_{k} x_{i j, k} v_{k}\right)^{2}
$$

When we differentiate $\mathrm{Q}$ with respect to each component $\mathrm{vk}$ and make the result equal to zero we drop into the equation

$$
\partial Q / \partial v_{k}=-2 \sum_{i=1}^{n} \sum_{j=1, j \neq i}^{n}\left(D_{i j}-\sum_{k} x_{i j, k} v_{k}\right)=0
$$

Equation (4) may be written in matrix form:

$$
\left(X^{t} X\right) v=X^{t} d
$$

where $\mathrm{X}$ is the matrix which represents the position of all taxons on the tree, $\mathrm{d}$ is the distance vector whose components are $\sum_{i=1}^{n} \sum_{j=1, j \neq i}^{n} D_{i j}$ and v stands for the branches tree.

The next step is to multiply both members of (5) by (X tX) -1 , then solve the so-called leastsquares equation for the length of the branches of this tree.

$$
v=\left(X^{t} X\right)^{-1} X^{t} d
$$

Put the linear system (6) in the form

$$
A v=f
$$


where in equation (7), $\mathrm{A}$ is a symmetric positive definite matrix $\left(\mathrm{n} \mathrm{x} \_\mathrm{n}, \mathrm{f}=\mathrm{X}\right.$ td is a vector associated to the independent term with dimension $\mathrm{n}$ and $\mathrm{v}$ is the unknown vector with dimension $\mathrm{n}$.

To solve the linear system (7) we could invert the matrix A and then use $A^{(-1)}=\left(L L^{t}\right)^{(-1)}=$ $\left(L^{t}\right)^{(-1)} L^{(-1)}$, where $\mathrm{L}$ is Cholesky decomposition lower triangular matrix $(\mathrm{n} \times \mathrm{n})$. This process becomes costly when $\mathrm{n}$ gets large, reaching the unsuitable threshold.

\section{NUMERICAL METHOD FOR SOLVING THE LINEAR SYSTEM}

Another way to solve linear system (7) is to take iterative methods that would fulfill required convergence criteria.

Being the matrix A positive definite, we can apply the Conjugate Gradient Method (CGM) to solve (7). Besides, the matrix A exhibits block profile, we can use iterative block-Jacobi as a preconditioner for the Preconditioned Conjugate Gradient Method (BPCGM) to solve (7), see Albrecht and Borges [3].

\subsection{GAUSS-JACOBI METHOD FOR BLOCK MATRICES}

Let us take the linear system (7) and we put the matrix A in the matrix block form:

$$
A=\left[\begin{array}{ccccccc}
B_{0} & F_{0} & \cdots & & \cdots & \cdots & \cdots \\
E_{1} & B_{1} & F_{1} & & \cdots & \cdots & \cdots \\
\vdots & \ddots & \ddots & & \vdots & \vdots & \vdots \\
\vdots & \vdots & \ddots & \ddots & & \ddots & \\
\cdots & \cdots & \cdots & E_{m-2} & B_{m-2} & F_{m-2} \\
\cdots & \cdots & \cdots & \cdots & E_{m-1} & B_{m-1}
\end{array}\right]
$$

where $\mathrm{Bk}, \mathrm{k}=0,1, \ldots \ldots, \mathrm{m}-1$, are diagonal blocks symmetric and positive definite (spd) matrices (nk $\mathrm{x} \mathrm{nk)}$.

We take $\mathrm{m}$ regular blocks matrices Bk, $(\mathrm{nk} \mathrm{x} \mathrm{nk}), \quad \sum_{k=0}^{m-1} n_{k}=n$.Thus, we have $\mathrm{m}-1$ rectangular

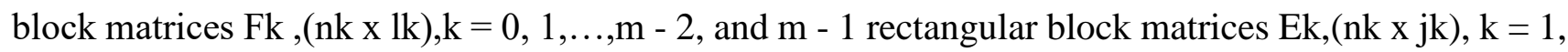
$2, \ldots, \mathrm{m}-1$.

One block Gauss-Jacobi iteration is defined by:

$$
\begin{gathered}
v_{0}{ }^{(i+1)}=-B_{0}^{-1} F_{0} v^{(i)}{ }_{(\text {right }) 0}+B_{0}^{-1} f_{0} \\
v_{k}{ }^{(i+1)}=-B_{k}^{-1} E_{k} v^{(i)}{ }_{(\text {left }) k}-B_{k}^{-1} F_{k} v^{(i)}{ }_{(r i g h t) k}+B_{k}^{-1} f_{k} \\
v_{m-1}{ }^{(i+1)}=-B_{m-1}^{-1} E_{m-1} v^{(i)}{ }_{(\text {left }) m-1}+B_{m-1}^{-1} f_{m-1}
\end{gathered}
$$


where $\mathrm{vk}$ and $\mathrm{fk}$ are the vectors of dimension $\mathrm{nk}, \mathrm{k}=0,1, \ldots, \mathrm{m}-1$ and $\mathrm{v}($ right $) \mathrm{k}, \mathrm{v}(\mathrm{left}) \mathrm{k}$ are the vectors of dimension lk and jk, respectively.

To calculate $B_{k}^{(-1)}$ we use $B_{k}^{-1}=\left(L_{k} L_{k}^{t}\right)^{-1}=\left(L_{k}^{t}\right)^{-1} L_{k}^{-1}$, where $L_{-} k$ is Cholesky decomposition lower triangular matrix (nk X nk).

\subsection{CONJUGATE GRADIENT METHOD}

To solve the linear system (7), we can use the conjugate gradient method (CGM).

This method consists to find the minimum of the quadratic function

$$
\phi(v)=\frac{1}{2} v^{t} A v-v^{t} f
$$

where $\mathrm{A}$ is a symmetric and positive definite (spd) matrix ( $\mathrm{x} \mathrm{n}$ ). The solution of the problem (9) correspond the solution of the problem (7).

Given tol: tolerance, $v^{0}=(1,1, \ldots, 1)^{t}$ and $\mathrm{N}=$ number of iterations, the Conjugate Gradient Method (CGM) can be given by the Algorithm 1:

$$
\begin{gathered}
\begin{array}{c}
\text { Figure 1: Algorithm for CGM } \\
r^{0}=A v^{\{0\}}-f \\
q^{\{0\}}=-r^{\{0\}} \\
\text { For } k=0,1,2, \ldots, N \\
\alpha=\frac{\left(r^{k}\right)^{t} r^{k}}{\left(q^{k}\right)^{t} A q^{k}} \\
v^{k+1}=v^{k}+\alpha q^{k} \\
/ * \text { Convergence Test */ } \\
\text { If abs }\left(v^{k+1}-v^{k}\right)<\text { tol, } \\
r^{k+1}=r^{k}+\alpha A q^{k} \\
\beta=\frac{\left(r^{k+1}\right)^{t} r^{k+1}}{\left(r^{k}\right)^{t} r^{k}} \\
q^{k+1}=-r^{k+1}+\beta q^{k}
\end{array}
\end{gathered}
$$

We know that in the worst case the convergence of the Conjugate Gradient Method (CGM) is obtained in $n$ steps. Since one iteration of CGM requires n2+O(n) operations, see Axelsson, Golub and Van Loan $[4,8]$, we deduce that in the worst case we need $n 3+O(n 2)$ operations. 


\subsection{PRECONDITIONED CONJUGATE GRADIENT METHOD}

The Conjugate Gradient Method (CGM) is proportional a $\sqrt{K(A)}$ ), where $\mathrm{K}(\mathrm{A})$ is the number condition of the matrix $\mathrm{A},\left(K(A)=\|A\|\|A\|^{-1}\right)$, see Axelsson, Golub and Van Loan $[4,8]$. One possibility to accelerate the convergence of the CGM applied to solve the system (7) preconditioning the matrix A and apply the Conjugate Gradient Method (CGM). Our problem allows to use the Block Gauss-Jacobi Method preconditioning the Conjugate Gradient Method. The matrix A is preconditioned by multiplication both terms of the equation (7) by $C^{-1} 1$, where $\mathrm{C}$ is a matrix composed by diagonals blocks matrices $B_{k}, \mathrm{k}=0,1, \ldots, \mathrm{m}-1$, are diagonal blocks symmetric and positive definite (spd) matrices (nk x nk). In this case, we solve the equivalent linear system:

$$
C^{-1} A v=C^{-1} f
$$

by the Conjugate Gradient Method (CGM). Since $\left.\mathrm{K}\left(C^{-1} A\right)<\mathrm{K}(\mathrm{A})\right)$, we use the block GaussJacobi preconditioning the Conjugate Gradient Method to solve the linear system (7). Given tol : tolerance, $v^{0}=(1,1, \ldots, 1)^{t} \quad$ and $\mathrm{N}=$ number of iterations, the Block Preconditioning Conjugate Gradient Method (BPCGM) can be given by the Algorithm 2:

$$
\begin{gathered}
\begin{array}{c}
\text { Figure 2: Algorithm for BPCGM } \\
r^{0}=A v^{0}-f \\
g^{0}=C^{-1} r^{0} \\
q^{0}=-g^{0} \\
\text { For } k=0,1,2, \ldots, N \\
\alpha=\left(r^{k}\right)^{t} g^{k} /\left(q^{k}\right)^{t} A q^{k} \\
v^{k+1}=v^{k}+\alpha q^{k} \\
\text { /* Convergence Test */ } \\
\text { Ifabs }\left(v^{k+1}-v^{k}\right)<\text { tol, } \\
r^{k+1}=r^{k}+\alpha A q^{k} \\
g^{k+1}=C^{-1} r^{k+1} \\
\beta=\left(r^{k+1}\right)^{t} r^{k+1} /\left(r^{k}\right)^{t} r^{k} \\
q^{k+1}=-g^{k+1}+\beta q^{k}
\end{array}
\end{gathered}
$$

The supplementary cost of the preconditioning conjugate gradient method is a construction of the sequence $g^{k}=C^{-1} r^{k}$. 


\section{RESULTS}

The problem to find a set of distances between taxons by using Distance Matrix Methods has been tested for some phylogenic trees. To solve the linear system (7) we used the algorithm 1 (CGM) and the algorithm 2 (BPCGM), with the total error norm $<10-5$. The matrix A, $\mathrm{n} \times \mathrm{n}$, in the linear system (7) is preconditioned by matrix $\mathrm{C}, \mathrm{nx}$, given by m block matrix $B_{k}, \mathrm{k}=0,1, \ldots, \mathrm{m}-1$.

Figure 3: Number of operations per thousand

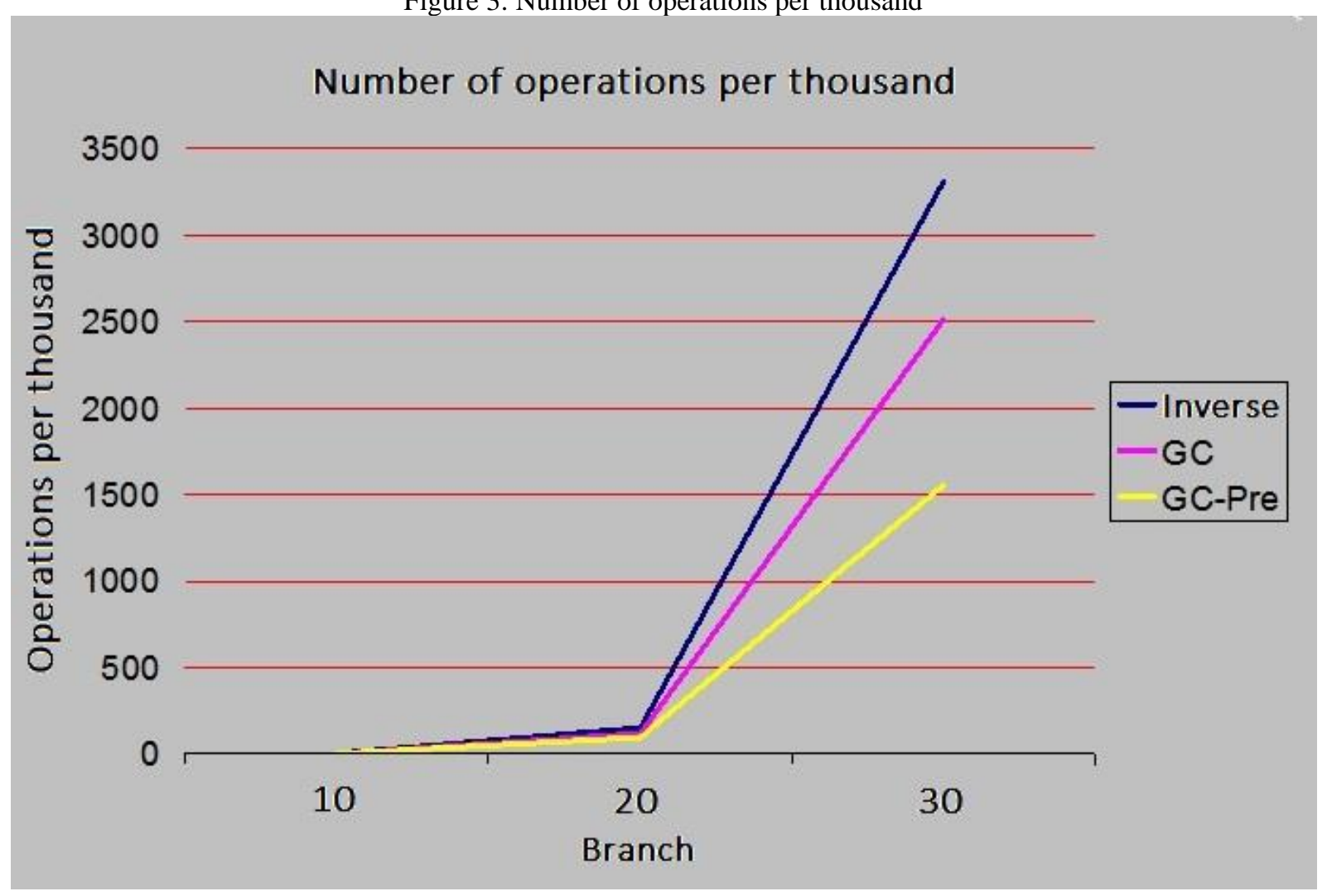

In Figure 3 we see the graph comparing the number of unknowns for solving the linear system (7) by the methods: inverse Cholesky, CGM and BPCGM.

\section{CONCLUSION}

We used the Conjugate Gradient Method (CGM) and Preconditioned Conjugate Gradient Method (BPCGM) with Block Jacobi as preconditioner. The preconditioned matrix C, $\mathrm{n}$ x_n, is shared in m different blocks matrix $B_{k}, \mathrm{k}=0,1, \ldots, \mathrm{m}-1$.

The dimension nk of the blocks $B_{k}$ has the influence in the convergence of (BPCGM), the convergence speed increases with the dimensions nk of the block matrix Bk.

The results confirm the equivalent number of operations of the system resolution by (CGM) and inverse Cholesky Method. 
To have one expressive gain using (BPCGM) for the system resolution in some cases, we have to share the matrix $\mathrm{C}, \mathrm{n} \times \mathrm{n}, \mathrm{m}$ different blocks matrix $B_{k}, \mathrm{k}=0,1, \ldots, \mathrm{m}-1$, with dimension nk not so small. Anyway, is conveniente to solve the linear system (7) by (CGM), simple to be implemented. 


\section{REFERENCES}

[1] Borges, N.; Medeiros. A. L. S. and Britto, M. M. A. - Método dos Mínimos Quadrados aplicado em Filogenia, XXXV CNMAC, Natal, RN, Brazil, 2014.

[2] Albrecht, F. F.; Borges, N. and Moura, C. A. - Phylogenetic Trees, Distance Matrices and Block Preconditioning Conjugate Gradient Method, ICIAM2011, Vancouver, 2011.

[3] Albrecht, F. F. and Borges, N. - Parallel methods for inferring phylogenies, PAMM Proc. Appl. Math. Mech., 7, pp. 2120007-2120008, 2007.

[4] Axelsson, O. - Iterative Solution Methods, Cambridge Univ. Press, 649 p., 1996.

[5] Cavalli-Sforza, L. and Edwards, A., - Phylogenetic analysis: models and estimation procedures, Am. J. Human Genetics, pp. 233-257, 1967.

[6] Felsenstein, J. - Inferring phylogenies, Massachusetts: Sinauer Associates, 580 p.,2003.

[7] Fitch, W.M. and Margoliash, E. - Construction of phylogenetic trees, Sci- ence, 760(157), pp. 279284, 1967.

[8] Golub,G. and Van Loan, C. - Matrix Computations, Johns Hopkins Univ. Press, 694 p., 1996.

[9] Keane,T. and al. - DPRml: distributed phylogeny reconstruction by maximum likelihood, Bioinformatics, 21(7), pp. 969-974 (2005).

[10] Stamatakis, A. - Distributed and parallel algorithms and systems for inference of huge phylogenic trees bases on the maximum likelihood method, Ph. D. thesis, 146 p., Technische Universitat Munchen, Germany, October 2004. 